\title{
Investigation of the correlation between norepinephrine transporter gene polymorphisms and essential hypertension
}

\author{
YING LI $^{1}$, LIQIANG ZHENG ${ }^{2}$, DINGYIN ZENG ${ }^{1}$, YING HAO $^{3}$, BAOGANG WU ${ }^{2}$ and YINGXIAN SUN ${ }^{1}$ \\ ${ }^{1}$ Department of Cardiac Diseases, the First Affiliated Hospital of China Medical University, Shen Yang, Liaoning 110001; \\ ${ }^{2}$ Sheng Jing Hospital of China Medical University, Shen Yang, Liaoning 110004; \\ ${ }^{3}$ Jin Qiu Hospital of Liaoning Province, Shen Yang, Liaoning 110015, P.R. China
}

Received February 12, 2012; Accepted May 31, 2012

DOI: $10.3892 / \mathrm{mmr} .2012 .1133$

\begin{abstract}
Essential hypertension (EH) is an etiological risk factor that poses a serious threat to human health. The aim of this study therefore was to investigate the correlation between the two SNPs (rs1805067 and rs2397771) of the norepinephrine (NE) transporter gene in the sympathetic nervous system and essential hypertension (EH). A total of 96 hypertensive patients (EH group) and 96 normal subjects (control group) were selected by epidemiological investigation and the sequences at rs1805067 and rs2397771 in the two groups were investigated using polymerase chain reaction (PCR) and Sanger sequencing. In the EH group, systolic and diastolic blood pressure, body mass index (BMI), levels of creatinine, low-density lipoprotein cholesterol (LDL-C), triglycerides (TG), and the percentage of drinkers were found to be higher compared with those of the control group $(\mathrm{P}<0.05)$. The results of the analysis of the allele frequencies of $\operatorname{rs} 1805067(\mathrm{~A} / \mathrm{G})$ revealed that there was no difference between the two groups $(\mathrm{P}>0.05)$. The analysis results of the allele frequencies of $\mathrm{rs} 2397771(\mathrm{C} / \mathrm{G})$ in the $\mathrm{EH}$ group revealed that $\mathrm{G}$ accounted for $48.96 \%$ and $\mathrm{C}$ for $51.04 \%$ of the patients, while in the control group, these values were 64.58 and $35.42 \%$, respectively. These results indicated a significant difference between the two groups $(\mathrm{P}<0.05)$. In the population studied, the onset of EH may be correlated with BMI, TG, LDL-C and high-density lipoprotein cholesterol (HDL-C). EH may be related to the rs2397771 (C/G) C allele polymorphism of the NE transporter gene but not to $\operatorname{rs} 1805067(\mathrm{~A} / \mathrm{G})$.
\end{abstract}

Correspondence to: Dr Yingxian Sun, Department of Cardiac Diseases, The First Affiliated Hospital of China Medical University, Shenyang, Nanjing North Street 155, District Heping, Shenyang, Liaoning 110001, P.R. China

E-mail: sunyingxian12@yahoo.com.cn

Key words: essential hypertension, norepinephrine transporter gene, gene polymorphism, rs1805067, rs2397771

\section{Introduction}

Essential hypertension (EH) is an etiological risk factor for a variety of cardiovascular and cerebrovascular diseases and is a serious threat to human health (1-3). The disease has become a major public health problem worldwide. $\mathrm{EH}$ is a polygenic disease, and the variation sites of each gene almost includes all the introns, exons and regulatory sequences (4).

Currently, over 150 genes that encode proteins have been found to be involved in the regulation of blood pressure via physiological, biochemical and metabolic pathways (5). These genes are termed candidate genes for EH. The genes associated with EH are mostly involved in the renin-angiotensinaldosterone system (RAAS), sympathetic nervous system, endothelial system and other signaling pathways. The enhanced reactivity of the sympathetic nerve is closely correlated with the onset of hypertension. The correlation between gene polymorphisms in the sympathetic nervous system and hypertension has been the subject of a number of studies (6). The norepinephrine (NE) transporter gene is a member of a large gene family of the sympathetic nervous system. Certain studies (7) have reported the existence of a correlation with hypertension, although such studies have not been performed in China. The aim of this study was to investigate the correlation between the two SNP sites (rs1805067 and rs2397771) of the NE transporter gene in the sympathetic nervous system and EH using polymerase chain reaction (PCR) and the Sanger sequencing method.

\section{Patients and methods}

Patients. Patients from the Fuxin City and Liaoning Province areas were selected for this study, based on the recommended diagnosis standard of hypertension in China in 2004. Selction of patients was based on criteria including systolic blood pressure (SBP) $\geq 140 \mathrm{mmHg}$, diastolic blood pressure (DBP) $\geq 90 \mathrm{mmHg}$ or patients who were ingesting antihypertensive agents. The screened patients, who were aged $\geq 18$ years and suffered from hypertension, were selected for an epidemiological survey, which included a general survey, history of cardiovascular disease, family history and living and eating habits. When filling in the questionnaire, the patients were directly questioned face-to-face by way of non-induced 
Table I. Comparison of data obtained by epidemiological survey from the hypertension and control groups.

\begin{tabular}{lccc}
\hline Variable & EH group $(\mathrm{n}=96)$ mean $\pm \mathrm{SD}$ & Control group $(\mathrm{n}=96)$ mean \pm SD & P-values \\
\hline Age $($ years $)$ & $38.35 \pm 6.549$ & $67.54 \pm 5.427$ & $<0.001$ \\
SBP $(\mathrm{mmHg})$ & $150.01 \pm 21.186$ & $126.22 \pm 10.236$ & $<0.001$ \\
DBP $(\mathrm{mmHg})$ & $98.24 \pm 13.64$ & $76.99 \pm 6.672$ & $<0.001$ \\
BMI $\left(\mathrm{kg} / \mathrm{m}^{2}\right)$ & $26.8536 \pm 3.59466$ & $21.8521 \pm 2.78093$ & $<0.001$ \\
TC $(\mathrm{mmol} / \mathrm{l})$ & $5.3340 \pm 0.98605$ & $5.2436 \pm 1.02181$ & $>0.05$ \\
HDL-C $(\mathrm{mmol} / \mathrm{l})$ & $1.3513 \pm 0.36568$ & $2.4978 \pm 0.37207$ & $<0.001$ \\
LDL-C $(\mathrm{mmol} / \mathrm{l})$ & $3.0481 \pm 0.75099$ & $0.1121 \pm 0.22973$ & $>0.05$ \\
TG & $0.2368 \pm 0.28285$ & $5.8758 \pm 1.76878$ & $<0.001$ \\
FPG $(\mathrm{mmol} / \mathrm{l})$ & $5.6478 \pm 1.33695$ & $53.850 \pm 12.2449$ & 50.05 \\
Serum creatinine $(\mu \mathrm{mol} / \mathrm{l})$ & $88.089 \pm 14.1849$ & $52.4 \%$ & $>0.001$ \\
Smokers $(\%)$ & $53.1 \%$ & $26.4 \%$ & $<0.05$ \\
Drinkers $(\%)$ & $48.8 \%$ & $<0.001$
\end{tabular}

BMI, body mass index; TC, total cholesterol; HDL-C, high density lipoprotein cholesterol; LDL-C, low density lipoprotein cholesterol; TG, triglyceride; FPG, fasting plasma glucose; SBP, systolic blood pressure; DBP, diastolic blood pressure; EH, essential hypertension.

inquiry. If the elderly patients were not able to answer the questions, the questionnaire was completed by their relatives. The selected patients provided informed consent. In the EH group, 96 patients were enrolled, 73 of whom were male and 23 female. In the control group, samples from nonhypertensive healthy individuals, aged $\geq 60$ years with blood pressure $<140 / 90 \mathrm{mmHg}$, were collected in the Fuxin City area. These healthy individuals completed the same epidemiological questionnaire. A total of 96 control subjects were enrolled, comprising 55 males and 41 females. The study was approved by the Ethics Committee of China Medical University.

\section{Research methods}

Physical examination. The blood pressure, height, weight and abdominal circumference of the patients and control subjects were measured.

Laboratory examinations. A 12-h fasting venous blood sample was collected from the subjects. One tube of blood was sent to the Mining Group General Hospital in Fuxin City, China, and examined with biochemical tests, including tests for liver and kidney function, fasting blood glucose, blood ion and blood lipids [total cholesterol (TC), triglyceride (TG), high-density lipoprotein cholesterol (HDL-C) and low-density lipoprotein cholesterol (LDL-C)]. A second tube of blood was used for the extraction of genomic DNA.

Genomic DNA extraction. DNA was extracted from the blood samples according to the manufacturer's instructions, and the concentration of the DNA was determined.

PCR assay. The primers were designed using Primer 5.0 primer design software and synthesized by Beijing Luhe Technology Co., Ltd. (Guangzhou, China). The sequences of the primers were: rs1805067 upstream, 5'-AGGATTCTAGGAGGACTGGGAGC-3' and downstream, 5'-TG GCGTCACCT TCAGCACTTT-3'; rs2397771 upstream, 5'-CCCCACCCAACCCACTTAG-3' and downstream, 5'-GAGACAGACCAAACGGGAAACA-3'.
A $25 \mu 1$ PCR assay system was used in this study, which contained $1 \mu \mathrm{l}$ genomic DNA, $2.5 \mu \mathrm{l}$ 10X Ex Taq buffer, $2 \mu \mathrm{l}$ $\operatorname{dNTP}(2.5 \mathrm{mmol} / \mathrm{l}), 0.5 \mu \mathrm{l}$ upstream primer $(10 \mathrm{mmol} / \mathrm{l}), 0.5 \mu \mathrm{l}$ downstream primer (10 mmol/l), $0.2 \mu \mathrm{l} \mathrm{Ex} \mathrm{Taq} \mathrm{and} 18.3 \mu \mathrm{l}$ $\mathrm{H}_{2} \mathrm{O}$. The conditions of the PCR assay were denaturation at $94^{\circ} \mathrm{C}$ for $5 \mathrm{~min}$, continued at $94^{\circ} \mathrm{C}$ for $40 \mathrm{sec}$, annealing at $54^{\circ} \mathrm{C}$ for $40 \mathrm{sec}$ and extension at $72^{\circ} \mathrm{C}$ for $60 \mathrm{sec}$ for 35 cycles, with a final extension at $72^{\circ} \mathrm{C}$ for $10 \mathrm{~min}$. PCR amplification was detected by agarose gel electrophoresis. Following electrophoresis, the results were observed using a gel imaging analysis system, the image was developed under appropriate exposure conditions and the plastic photographic map was saved. The PCR products were purified and then sequenced using a $5 \mu \mathrm{l}$ system of sequencing reaction including $2 \mu \mathrm{l}$ template, $1 \mu \mathrm{l}$ primer and $2 \mu \mathrm{lBD}$. The reaction system was sequenced using the ABI 3730 sequencer.

Statistical analysis. Results were analyzed using statistical SPSS 13.0 software (SPSS, Inc., Chicago, IL, USA) and the continuous data were shown as the mean \pm standard deviation. The comparison of the two independent samples was performed using the t-test. The qualitative data were compared using the $\chi^{2}$ test. The goodness-of-fit of genetic balance was tested using the Hardy-Weinberg law of genetic equilibrium.

\section{Results}

Analysis of epidemiological data. In the EH group, SBP $(\mathrm{P}<0.001)$, DBP $(\mathrm{P}<0.001)$, body mass index (BMI; $\mathrm{P}<0.001)$, creatinine $(\mathrm{P}<0.001)$, LDL-C $(\mathrm{P}<0.05), \mathrm{TG}$ in serum $(\mathrm{P}<0.001)$ and the percentage of drinkers $(\mathrm{P}<0.001)$ of the patients were found to be higher than those in the control group (Table I). The number of individuals of an advanced age $(\mathrm{P}<0.001)$ and the HDL-C levels $(\mathrm{P}<0.001)$ in the $\mathrm{EH}$ group were lower than those of the control group. No significant difference was found between the total protein, albumin, TC, fasting plasma glucose 
A

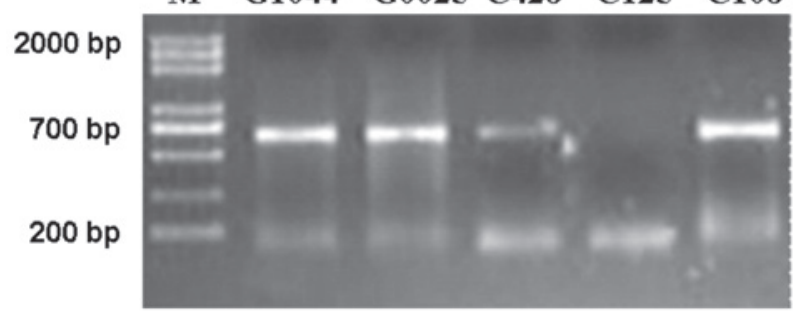

B

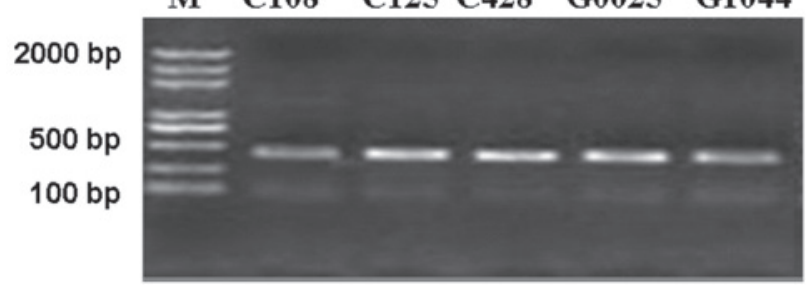

Figure 1. Amplified products of the two sites (rs1805067 and rs2397771) of norepinephrine transporter genes by PCR. The amplified objective fragments of rs1805067 and rs2397771 are (A) $640 \mathrm{bp}$ and (B) $437 \mathrm{bp}$, respectively. $\mathrm{M}$, marker; $\mathrm{C} 108, \mathrm{C} 125$ and $\mathrm{C} 428$ are three samples from the control group; G0025 and G0044 are two samples from the essential hypertension (EH) group.

(FPG) and the percentage of smokers in the EH and control groups $(\mathrm{P}>0.05)$.

PCR electrophoresis product of the two sites of NE transporter gene rs 1805067 and rs2397771. Samples were amplified by PCR with the upstream and downstream primers (Fig. 1). The fragment of the target gene rs1805067 was $640 \mathrm{bp}$ and rs2397771 was 437 bp.

$N E$ transporter gene $r s 1805067(A / G)$. The analysis of the allele frequencies of rs $1805067(\mathrm{~A} / \mathrm{G})$ in the EH and control groups revealed that $\mathrm{G}$ accounted for 100 and $\mathrm{A}$ for $0 \%$ of the subjects. No difference was found between the two groups $\left(\chi^{2}=0, P>0.05\right)$. The genotypes of the subjects in the two groups were GG homozygous and the polymorphisms were not found. For the control group, the Hardy-Weinberg law of genetic equilibrium was adopted to detect the genotype distribution, but no genetic imbalance was found $(\mathrm{P}=0.00)$. The sequences of the EH and control groups are shown in Fig. 2A and $\mathrm{B}$, respectively, and the allele frequencies and genotype distribution are shown in Table II.

The NE transporter gene $r s 2397771(C / G)$. The analysis of the allele frequencies of $\mathrm{rs} 2397771(\mathrm{C} / \mathrm{G})$ in the EH group revealed that $\mathrm{G}$ accounted for $48.96 \%$ and $\mathrm{C}$ for $51.04 \%$ of the individuals, whereas in the control group, these values were 64.58 and $35.42 \%$, respectively. A significant difference was observed between the two groups $\left(\chi^{2}=4.7751\right.$, $\mathrm{P}=0.0289, \mathrm{P}<0.05)$ and the odds ratio $(\mathrm{OR})$ was found to be 1.9011 [95\% confidence interval (CI), 1.0657-3.3916). With regard to genotypes, in the $\mathrm{EH}$ group, $\mathrm{CC}$ accounted for 12.5, GG for 39.58 and CG for $47.92 \%$ of the individuals, while in the control group these values were 8.33, 47.92 and $43.75 \%$, respectively. No significant difference was found between the two groups $\left(\chi^{2}=1.7437, \mathrm{P}=0.4182, \mathrm{P}>0.05\right)$. For the control group, the Hardy-Weinberg law of genetic equilibrium was adopted to detect the genotype distribution. As $\mathrm{P}=0.348$ ( $P>0.05)$, it reached genetic balance, suggesting the group was representative. The sequences of the $\mathrm{EH}$ and control groups are shown in Fig. 2C and D, respectively, and the allele frequencies and genotype distribution are shown in Table III.

\section{Discussion}

$\mathrm{NE}$ is a catecholamine neurotransmitter, which is found in the postganglionic sympathetic and central nervous systems (8). In noradrenergic neurons, synaptic transmission includes three steps: NE is released from the presynaptic membrane, $\mathrm{NE}$ in the synaptic cleft gap acts on postsynaptic membrane
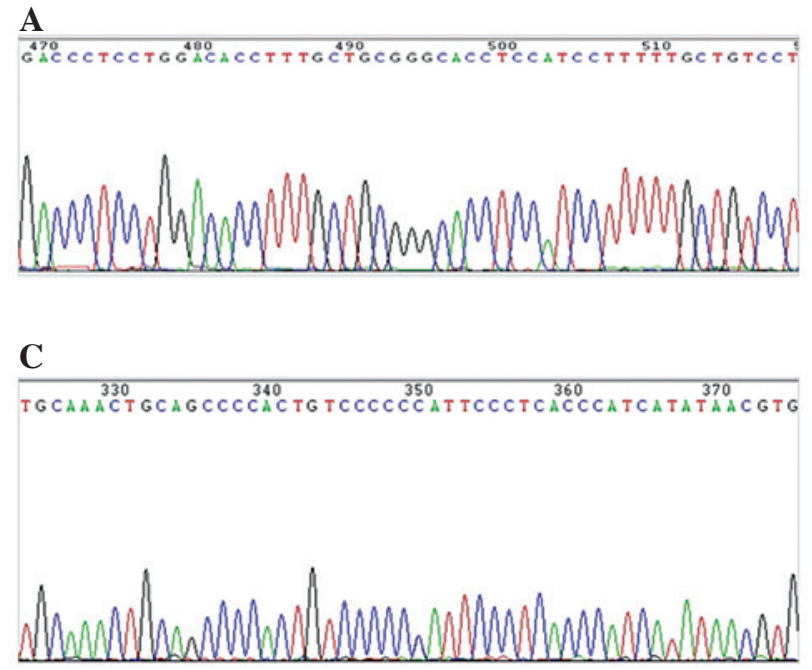

B

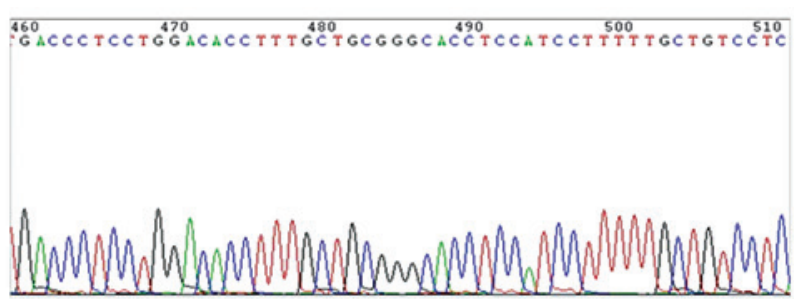

D

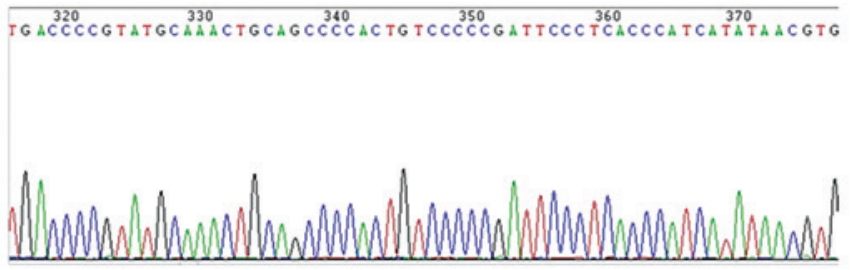

Figure 2. Sequencing figures of the two sites (rs1805067 and rs2397771) of the norepinephrine transporter gene of the patients in the EH and the control groups. The sequencing figure of (A) rs1805067 (A/G) polymorphism in the EH group; (B) rs1805067 (A/G) polymorphism in the control group; (C) rs2397771 (C/G) polymorphism in the EH group and (D) rs2397771 (C/G) polymorphism in the control group. EH, essential hypertension. 
Table II. Allele frequency and genotype distribution of rs1805067 (A/G) site, n (\%).

\begin{tabular}{lcccccc}
\hline Group & Case & Allele A & Allele G & Genotype AA & Genotype GG & Genotype AG \\
\hline EH group & 96 & $0(0)$ & $96(100)$ & $0(0)$ & $96(100)$ & $0(0)$ \\
Control group & 96 & $0(0)$ & $96(100)$ & $0(0)$ & $96(100)$ & $0(0)$ \\
$\chi^{2}$ & & 0 & & & 0 & $>0.05$ \\
P-value & & $>0.05$ & & & \\
\hline
\end{tabular}

EH, essential hypertension.

Table III. Allele frequency and genotype contribution of rs2397771 (C/G) site, n (\%).

\begin{tabular}{|c|c|c|c|c|c|c|}
\hline Group & Case & Allele C & Allele G & Genotype CC & Genotype GG & Genotype CG \\
\hline EH group & 96 & $49(51.04)$ & 47 (48.96) & $12(12.5)$ & $38(39.58)$ & $46(47.92)$ \\
\hline Control group & 96 & $34(35.42)$ & $62(64.58)$ & $8(8.33)$ & 46 (47.92) & $42(43.75)$ \\
\hline$\chi^{2}$ & & 4.7751 & & 1.7437 & & \\
\hline P-value & & 0.0289 & & 0.4182 & & \\
\hline
\end{tabular}

GG:CC $\chi^{2}=1.4101, \mathrm{P}=0.2350, \mathrm{OR}=1.8158$ (95\% CI, 0.6731-4.8986); CG:CC $\chi^{2}=0.3914, \mathrm{P}=0.5316, \mathrm{OR}=1.3696$ (95\% CI, 0.5101-3.6768). $\mathrm{EH}$, essential hypertension; OR, odds ratio; CI, confidence interval.

receptors and NE is reabsorbed by presynaptic neurons or glial cells. The reuptake process of NE requires the involvement of the sodium-dependent NE transporter (NET). NET is located in the presynaptic adrenergic nerve membrane and belongs to the family of monoamines (including NE, dopamine and serotonin), which are sodium and chlorine-dependent transport proteins. The function of NET is to reuptake NE released from the neuron back to the presynaptic membrane, which is crucial in the regulation of NE concentration in the synaptic cleft, termination of nerve impulse signals and maintenance of the sensitivity of neurotransmitter receptors. NET, as a significant regulator of adrenergic neurotransmitters and the neurotransmitter receptor signaling pathway, is important in regulating the functions of the cardiovascular system (9).

An abnormal increase in sympathetic activity is a significant pathogenesis of hypertension. In a study by Schlaich et al (10), electrical impulses from the postganglionic sympathetic sural nerve were recorded using a micro-electrical nerve. NE labeled with ${ }^{3} \mathrm{H}$ was intravenously injected and the NE kinetics of healthy individuals and patients with hypertension were determined by isotope dilution. The systemic and local (heart and kidney) NE release rates were determined following the administration of the NET inhibitor desipramine. The plasma levels of 3,4-dihydroxyphenylglycol (DHPG) in the NE neurons and 3-methoxy-4-hydroxyphenylglycol (MHPG) outside the NE neurons were simultaneously detected. The results showed that the electrical activity of the sympathetic nerve in hypertensive patients was increased compared with that in healthy individuals and the release of systemic and cardiac NE were also increased. The level of DHPG was reduced, while the level of MHPG was increased. The reduction of NE reuptake and the function of NET reuptake are important in enhancing sympathetic activity, but the mechanism by which this occurs is not clear and the correlation between the functional decline of the NET gene and polymorphism change of the NET gene was not confirmed (10).

The human NET gene SLC6A2 is located at chromosome $16 q 12.2$ and its length is $45 \mathrm{~kb}$. The gene contains 14 unevenly distributed exons, whose lengths are between 60 and $400 \mathrm{bp}$ and the lengths of the introns vary widely from 0.2 to $13.2 \mathrm{~kb}$. The exon was found to be significantly correlated with the transmembrane functional area of the protein. In the process of transcription, different splicing of the exons results in three variants of protein with different carboxyl terminals, hNET, hNET $\mathrm{Ct}$ var1 and hNet $\mathrm{Ct}$ var2. Of these variants, hNET and hNET Ct var2 are involved in transcription, although the detailed tissue distribution of the three variants is not yet clear (11). Few studies concerning NET gene polymorphisms have been performed. Rumantir et al (12) detected the reduction of NE reuptake in the neurons of a hypertensive population using the radioactive tracing method. Ono et al (13) found 13 polymorphism sites in the SLC6A2 gene of a Japanese population comprising 1,950 individuals in 2003. This was the first study to report that the polymorphisms in the promoter $3 \mathrm{~A} / \mathrm{G}$ are correlated with hypertension in the relevant Japanese population and the distribution frequencies of the $A G$ and GG genotypes in the Japanese hypertensive population were higher. In a study by Liu et al (14), the results of animal experiments suggested that the abnormal expression of NET may be caused by heart failure based on cardiac sympathetic nerve dysfunction. In 2004, Schlaich et al (10) found that the Gly478Ser polymorphism in the NET gene was significantly correlated with hypertension and suggested that the Gly478Ser polymorphism may lead to a decrease in the affinity of NE and NET in the synaptic gap.

No study concerning the correlation between the SLC6A2 polymorphism and hypertension in China has been performed 
previously. In this study, rs1805067 and rs2397771, the two SNP sites of the SLC6A2 gene, were amplified by PCR and then adopted with Sanger sequencing to evaluate their correlation with hypertension. The results showed that the rs1805067 site $(A / G)$ had no significant correlation with hypertension. In the Liaoning province of China, this locus may be homozygous and no polymorphism was found, which was inconsistent with the results of studies from other countries. This observation indicates that there are ethnic and regional differences in the polymorphism at this site. Previous studies revealed that the polymorphism of rs2397771 (C/G) may be associated with heart failure and depression, but its correlation with hypertension has not been reported. Our results indicate that the allele frequency of $\mathrm{rs} 2397771(\mathrm{C} / \mathrm{G}) \mathrm{C}$ may be associated with hypertension. With regard to genotype distribution, no significant difference was found between the two groups. However, these results require further validation in a clinical trial with a larger sample size.

\section{Acknowledgements}

This study was supported by a grant from the Special Program for Key Basic Research of the Ministry of Science and Technology, National program on key basic research project: 973 program China (no. 2010CB535011).

\section{References}

1. Mitchell JR: Hypertension and arterial disease. Br Heart J 33: 122-126, 1971.

2. Muiesan G: Risk factors in hypertension and ischaemic heart disease. Postgrad Med J 3: 15-20, 1979.

3. Kannel WB and Gordan T: Evaluation of cardiovascular risk in the elderly: the Framingham study. Bull N Y Acad Med 54: 573-591, 1978
4. Weder AB: Genetics and hypertension. J Clin Hypertens (Greenwich) 9: 217-223, 2007.

5. Armani C, Botto $\mathrm{N}$ and Andreassi MG: Susceptibility genes in hypertension. Curr Pharm Des 17: 2973-2986, 2011.

6. Wang JG and Staessen JA: Genetic polymorphisms in the reninangiotensin system: relevance for susceptibility to cardiovascular disease. Eur J Pharmacol 410: 289-302, 2000.

7. Bonisch $\mathrm{H}$ and Bruss $\mathrm{M}$ : The norepinephrine transporter in physiology and disease. Handb Exp Pharmacol 175: 485-524, 2006.

8. Kruk ZL and Pycock CJ (eds): Neurotransmitters and Drugs. 3rd edition. Chapman \& Hall Press, London, 1992.

9. Liu X, Li J, Shi J, et al: The research advancement of arterenol levaterenol transport protein. Med J Chin People's Armed Police Forces 16: 294-296, 2005 (In Chinese).

10. Schlaich MP, Lambert E, Kaye DM et al: Sympathetic augmentation in hypertension role of nerve firing, norepinephrine reuptake, and angiotensin neuromodulation. Hypertension 43: 169-175, 2004.

11. Torres GE, Gainetdinov RR and Caron MG: Plasma membrane monoamine transporters: structure, regulation and function. Nat Rev Neurosci 4: 13-25, 2003.

12. Rumantir MS, Kaye DM, Jennings GR, et al: Phenotypic evidence of faulty neuronal norepinephrine reuptake in essential hypertension. Hypertension 36: 824-829, 2000.

13. Ono K, Iwanaga Y, Mannami T, et al: Epidemiological evidence of an association between SLC6A2 gene polymorphism and hypertension. Hypertens Res 26: 685-689, 2003.

14. Liu X, Li H and Cui G: Alteration of cardiac sympathetic norepinephrine transporter expression in rats with experimental heart failure induced by long-term pressure overload. Chin J Pathophysiol 23: 601-603, 2007 (In Chinese). 